\title{
Effect of Combining Multiple CNV Defining Algorithms on the Reliability of CNV Calls from SNP Genotyping Data
}

\author{
Soon-Young $\mathrm{Kim}^{1,2}$, Ji-Hong Kim ${ }^{1,2}$, Yeun-Jun Chung ${ }^{1,2 *}$ \\ ${ }^{1}$ Integrated Research Center for Genome Polymorphism, The Catholic University of Korea School of Medicine, Seoul 137-701, \\ Korea, ${ }^{2}$ Department of Microbiology, The Catholic University of Korea School of Medicine, Seoul 137-701, Korea
}

\begin{abstract}
In addition to single-nucleotide polymorphisms (SNP), copy number variation (CNV) is a major component of human genetic diversity. Among many whole-genome analysis platforms, SNP arrays have been commonly used for genomewide CNV discovery. Recently, a number of CNV defining algorithms from SNP genotyping data have been developed; however, due to the fundamental limitation of SNP genotyping data for the measurement of signal intensity, there are still concerns regarding the possibility of false discovery or low sensitivity for detecting CNVs. In this study, we aimed to verify the effect of combining multiple CNV calling algorithms and set up the most reliable pipeline for CNV calling with Affymetrix Genomewide SNP 5.0 data. For this purpose, we selected the 3 most commonly used algorithms for CNV segmentation from SNP genotyping data, PennCNV, QuantiSNP; and BirdSuite. After defining the CNV loci using the 3 different algorithms, we assessed how many of them overlapped with each other, and we also validated the CNVs by genomic quantitative PCR. Through this analysis, we proposed that for reliable CNV-based genomewide association study using SNP array data, CNV calls must be performed with at least 3 different algorithms and that the CNVs consistently called from more than 2 algorithms must be used for association analysis, because they are more reliable than the CNVs called from a single algorithm. Our result will be helpful to set up the CNV analysis protocols for Affymetrix Genomewide SNP 5.0 genotyping data.
\end{abstract}

Keywords: CNV defining algorithm, DNA copy number variations, SNP array

\section{Introduction}

Human genome variation has facilitated the understanding of inter-individual phenotypic differences $[1,2]$. In addition to single-nucleotide polymorphisms (SNPs), it is widely accepted that large-scale DNA structural variation, termed copy number variation (CNV), is a major component of human genetic diversity $[2,3]$. Genomewide SNP genotyping data can be used for $\mathrm{CNV}$ calling [4]; therefore, if we can get reliable CNV calls from the SNP genotyping data, a CNV-based genomewide association study (GWAS) can be realized. Among many whole-genome $\mathrm{CNV}$ analysis platforms, SNP arrays have been suggested as a resource for $\mathrm{CNV}$ discovery due to their ubiquitous genome coverage and relatively advantageous resolution. However, despite the importance of $\mathrm{CNV}$-disease association analysis, $\mathrm{CNV}$ calling from SNP genotyping data has not been well established. Affymetrix Genomewide SNP 5.0 is one of the commonly used SNP array platforms for SNP-GWAS as well as $\mathrm{CNV}$ analysis [5]. We previously validated the accuracy and reproducibility of CNVs called from Affymetrix SNP array 5.0 data by comparing the $\mathrm{CNV}$ calls from 3 different array platforms using NEXUS software: Affymetrix SNP array 5.0, Agilent $2 \mathrm{X} 244 \mathrm{~K} \mathrm{CNV}$ array, and NimbleGen $2.1 \mathrm{M}$ CNV array [6].

Recently, a number of CNV defining algorithms have been developed, which have facilitated the CNV-based GWAS [7-14]. However, due to the fundamental limitation of SNP genotyping data for the measurement of signal intensity, there are still concerns regarding the possibility of false discovery or low sensitivity for detecting CNVs $[15,16]$. Indeed, $\mathrm{CNV}$ calling is dependent on the types of array 
platforms and analytic tools. Each platform and calling algorithm has its own advantages and disadvantages; so, one single algorithm or array platform is not always best for determination of CNVs [17-19]. Recently Pinto et al. [20] showed that different analytic tools applied to the same raw data typically yielded CNV calls with $<50 \%$ concordance and, using multiple algorithms, minimize the number of false discoveries. To remedy the potential limitations of SNP array for $\mathrm{CNV}$ detection, more than one way of $\mathrm{CNV}$ calling by using several different segmentation algorithms are performed, and overlapped calls are used for GWAS analysis [21-24].

In this study, we tried to verify the effect of adopting multiple CNV calling algorithms and set up the most reliable pipeline for CNV calling with Affymetrix Genomewide SNP 5.0 data. We selected the 3 most commonly used algorithms for CNV segmentation from SNP genotyping data, PennCNV, QuantiSNP, and BirdSuite. After defining the CNV loci using the 3 different algorithms, we assessed how many of them overlapped with each other, and we also validated the CNVs by genomic quantitative PCR (qPCR). Finally we concluded that CNVs that were consistently called from more than 2 different calling algorithms are more reliable than the CNVs called from a single algorithm. Our result will be helpful to set up the CNV analysis protocols for Affymetrix Genomewide SNP 5.0 genotyping data.

\section{Methods}

\section{Study materials}

We used Affymetrix Genomewide SNP 5.0 genotyping data provided by the Korea Association Resource (KARE) consortium, Korean Genome Epidemiology Study (KoGES). As a control, we purchased a HapMap cell line, GM10851, from Coriell Institute for Medical Research (Camden, NJ, USA) and extracted genomic DNA using the DNeasy Blood \& Tissue Kit (Qiagen, Hilden, Germany).

\section{Pre-processing SNP array data}

Before $\mathrm{CNV}$ calling procedures, all required pre-processing procedures, including allele correction, summarization, and background correction, were performed as described previously by using the software provided by Affymetrix [5]. In brief, background-corrected data were normalized using quantile normalization and summarized by median polish.

\section{CNV calling}

For this study, Affymetrix 5.0 SNP array (Affymetrix, Santa Clara, CA, USA) data of 10 subjects were randomly selected from the KARE dataset for CNV calling. Affymetrix
5.0 data of NA10851 was used as a control. For defining CNVs, we choose 3 different segmentation algorithms, PennCNV [25], QuantiSNP [26] and BirdSuite [27]. PennCNV implements a hidden Markov model (HMM) and considers SNP allelic ratio distribution in addition to signal intensity. We only used CNVs from PennCNV with samples that had a standard deviation of $\log \mathrm{R}$ ratio (LRR) smaller than 0.2, drift values of b-allele frequency (BAF_drift) smaller than 0.01, and waviness factor between -0.05 and 0.05 . For QuantiSNP, we did not apply any criteria for sample filtering, since QuantiSNP uses an Objective Bayes HiddenMarkov Model (OB-HMM) for calibrating the model for fixing the false positive error rate and maximum marginal likelihood to set other hyperparameters. It was originally developed for detecting CNVs from BeadArray SNP genotyping data of Illumina, but Affymetrix data also could be processed as long as they have probe signal intensities and B-allele frequencies. Birdsuite uses exclusive copy number analysis routine (Canary) for CNP locus and HMM for finding rare $\mathrm{CNVs}$. It generates a logarithm of the odds ratio (LOD) value for each $\mathrm{CNV}$ that describes the likelihood of a $\mathrm{CNV}$ relative to no $\mathrm{CNV}$ over a given interval, including flanking sequences. Of the BirdSuite calls, only the CNVs that have an LOD value smaller than 2 were used for further analysis. All 3 methods were used with default values, and no other option was added or changed.

\section{Validation}

To estimate the false discovery rate of our CNV-calling algorithm, CNVs were validated by genomic real-time qPCR. For this purpose, we randomly selected $25 \mathrm{CNV}$ loci and performed genomic qPCR using DNAs from study subjects who showed corresponding CNVs on that loci. The PCR primers used in this study were designed using the PrimerQuest program (http://www.idtdna.com/Scitools/ Applications/Primerquest). To verify the specificity of the $\mathrm{PCR}$ reactions under the unified denaturation temperature $\left(60^{\circ} \mathrm{C}\right)$, we performed PCR and agarose gel electrophoresis for each primer set. We also screened the University of California Santa Cruz (UCSC) database (http://genome. ucsc.edu/) to confirm the unique sequence without any repeat sequences in the primers. Sequence information of the primers for genomic qPCR validation is listed in Table 1.

Ten microliters of the reaction mixture contained $10 \mathrm{ng}$ of genomic DNA, SYBR Premix Ex Taq TM II (TaKaRaBio, Shiga, Japan), $1 \times$ ROX (Toyobo, Osaka, Japan), and 10 pmol of primers. Thermal cycling conditions consisted of 1 cycle of $10 \mathrm{~s}$ at $95^{\circ} \mathrm{C}$, followed by 40 cycles of $5 \mathrm{~s}$ at $95^{\circ} \mathrm{C}, 10 \mathrm{~s}$ at $61^{\circ} \mathrm{C}$, and $20 \mathrm{~s}$ at $72^{\circ} \mathrm{C}$. All PCR experiments were repeated twice, and amplification efficiencies for both target and reference genes were evaluated using a standard curve over $1: 5$ serial 
Table 1. Sequence information of the primers for genomic qPCR validation

\begin{tabular}{|c|c|c|c|c|c|}
\hline Chr & Start & End & Length (bp) & Forward & Reverse \\
\hline 1 & $108,538,343$ & $108,538,461$ & 119 & AGGAGGTTGCACCATGGTTAGTCA & GGCCACAGCACATCTTGTGAAACA \\
\hline 1 & $150,842,026$ & $150,842,185$ & 160 & AACCATGGACTTCGTGGGTAGTCA & САTGСТССАTGCATTGTGGTGGAA \\
\hline 3 & 259,837 & 259,952 & 116 & CAAATGGAACAGCAGGGTCAGCAA & TGCTGTGTCCAGCATCCTATGTGT \\
\hline 4 & $69,073,047$ & $69,073,213$ & 167 & TTGTTGGAGGAACAAAGCCCAACC & TGGCTGGTGTCTGTTCTGATTGGT \\
\hline 5 & $92,610,728$ & $92,610,891$ & 164 & AGGTTGATGAGCCACACAGGGTAT & TGСТССТGААТTССТСАGСТTССА \\
\hline 5 & $178,045,652$ & $178,045,850$ & 199 & AGGCAAGAGGTAGCCCACCTTAAT & AAAGCAGGAGCTGAGAGGCAGAAA \\
\hline 7 & $141,704,582$ & $141,704,737$ & 156 & AAACAGACAGGCACTGGTCCATCT & ATGGCATAACСТССАТСССАСТСА \\
\hline 7 & $141,711,867$ & $141,712,039$ & 173 & TGAGACTGTGGATCTTTGGCCACT & TAATTCCACATGTCCAGGCCCACT \\
\hline 7 & $154,025,113$ & $154,025,228$ & 116 & TGCAATGGCACGATCTTGTCTCAC & AGGCATGATGGTGGGTGCCTATAA \\
\hline 11 & $55,162,545$ & $55,162,631$ & 87 & TCTATCACGTGCACCCAGCTCATT & TGTGGATGTGTAGCAAAGGTCGGA \\
\hline 11 & $55,207,777$ & $55,207,961$ & 185 & TGCACТАСАССАТСАТСАСGАССА & ATCAATGCGAGCCAACTTCAGCAG \\
\hline 14 & $81,569,884$ & $81,569,974$ & 91 & TGCATGTTAGGAGGCTGTGGATCA & TGAGGCAGAACAATGTGGCCTCTA \\
\hline 14 & $81,569,895$ & $81,569,974$ & 80 & AGGCTGTGGATCAATACGGGTTCA & TGAGGCAGAACAATGTGGCCTCTA \\
\hline 16 & $54,363,707$ & $54,363,878$ & 172 & AGCCGCATCTGTAGTCCTGAAAGT & GTTCССТCCAAAGCTGGCAATGTT \\
\hline 5 & 814,195 & 814,318 & 124 & ACCTCGGCCGGATTCTGGATTAAA & ACCTTCATGGCAGGTGAGAAGACA \\
\hline 9 & $44,685,893$ & $44,685,973$ & 81 & ATGACAGACAGGACCCAACACCAT & TCAACAATAGGGCAGAGGAAGCCA \\
\hline 15 & $19,145,532$ & $19,145,684$ & 153 & GGCGCAGTGGTTCATGCTTGTAAT & TGCGTACCACCATGCCTAGCTAAA \\
\hline 15 & $19,833,989$ & $19,834,167$ & 179 & TGCCTAAGCTGTGTTACTCTGCCA & CGCAAAGGGTTACAGATGGCAACA \\
\hline 20 & $1,531,337$ & $1,531,532$ & 196 & ATCACCCAATTGCGGACTCСТCTT & ACAGACTCTACGGCGTTGGCTTTA \\
\hline 22 & $22,702,643$ & $22,702,799$ & 157 & GCCTGACTTCGAAATGGTGGCAAA & TGGTTGCCTGGTTTCTAGCCСTAT \\
\hline 2 & $87,557,596$ & $87,557,722$ & 127 & TGAGAGGCAGGTGGATTTGGATGT & TGGAAGACACACAGCGAACCTCTT \\
\hline 4 & $70,207,254$ & $70,207,437$ & 184 & АССТСАААTTTCAGTGCCGAAGGC & тCTTCTGTGCTGGCTGTGGATTCT \\
\hline 15 & $22,237,790$ & $22,237,886$ & 97 & AGCTCAGGAGATGAAAGGGCACAT & TCTGCСTGAAGCAAGTGTACCTGT \\
\hline 15 & $19,843,004$ & $19,843,164$ & 161 & AGACTTGCCTTCTTGTCAGCCTCA & AGACAGGGCAGGAAGAACTTTCCA \\
\hline 19 & $48,112,193$ & $48,112,355$ & 163 & TCСТGСАТССТССТGTGTGАСАTT & TGGCTACATCTGGTACAAAGGGCA \\
\hline
\end{tabular}

qPCR, quantitative PCR; Chr, chromosome.

Table 2. General characteristics of the copy number variation (CNV) calls from 3 algorithms

\begin{tabular}{|c|c|c|c|c|c|c|c|}
\hline & \multicolumn{3}{|c|}{ No filtration } & \multicolumn{3}{|c|}{$>7$ consecutive proves } \\
\hline & & PennCNV & QuantiSNP & BirdSuite & PennCNV & QuantiSNP & BirdSuite \\
\hline \multicolumn{2}{|c|}{ Total no. of calls } & 814 & 1,087 & 1,362 & 295 & 311 & 385 \\
\hline \multirow[t]{2}{*}{ Size } & Average & $44,672.6$ & 39,911 & $15,466.5$ & 104,264 & 103,782 & $34,463.9$ \\
\hline & Median & 12,981 & 7,599 & 7,964 & 50,799 & 50,597 & 23,528 \\
\hline Small CNV & $<1 \mathrm{kbp}$ & $49(6.0 \%)$ & $272(25.0 \%)$ & $193(14.2 \%)$ & 0 & 0 & 0 \\
\hline
\end{tabular}

dilutions. The copy number of each target was defined as 2$\Delta \Delta \mathrm{CT}$, where $\Delta \mathrm{Ct}$ is the difference in threshold cycles for the sample in question normalized against the reference gene (RNaseP) and expressed relative to the value obtained by calibrator DNA (NA10851 and Promega DNA), as described elsewhere [28].

\section{Results and Discussion}

The general characteristics of the CNV calls from the 3 algorithms are shown in Table 2. The total number of CNV calls from the 3 algorithms was similar-range 814 to 1,362 . The median size of the CNVs called by PennCNV $(12.9 \mathrm{~kb})$ were bigger than the other 2 algorithms $(7.5 \mathrm{~kb}$ and $7.9 \mathrm{~kb})$.
The size of the CNVs consistently detected by 2 or more algorithms was generally bigger than that of the CNVs detected by a single method. For example, the average sizes of the CNVs that were detected from all 3 algorithms, 2 algorithms, and 1 method were $107 \mathrm{~kb}, 73.2 \mathrm{~kb}$, and $15.9 \mathrm{~kb}$, respectively (Table 3 ). Fig. 1 shows the examples of different sizes of CNVs according to the LRR distribution of gain- and loss-type $\mathrm{CNV}$ regions, depending on how many algorithms detected the CNV in that region. These data suggest that larger-sized CNVs are generally more prominent; so, they can be relatively easily detected by any segmentation method, regardless of the algorithm. But, detection of the smaller-sized CNVs can be affected more easily by the characteristics of the $\mathrm{CNV}$ defining algorithm; so, they can 
Table 3. Number of copy number variations (CNVs) consistently defined from 3 different algorithms

\begin{tabular}{|c|c|c|c|c|c|c|c|c|c|c|}
\hline & \multicolumn{5}{|c|}{ No filtration } & \multicolumn{5}{|c|}{$>7$ consecutive proves } \\
\hline & Size & $\mathrm{CNV}$ & PennCNV & QuantiSNP & BirdSuite & Size & CNV & PennCNV & QuantiSNP & BirdSuite \\
\hline All overlap & 107.2 & 40 & 40 & 42 & 40 & 208 & 14 & 14 & 15 & 15 \\
\hline 2 overlap & 73.2 & 425 & 356 & 424 & 91 & 130 & 231 & 212 & 223 & 14 \\
\hline Unique & 15.9 & 2,270 & 418 & 621 & 1,231 & 40 & 498 & 69 & 73 & 356 \\
\hline
\end{tabular}

Size: average size $(\mathrm{kb})$.
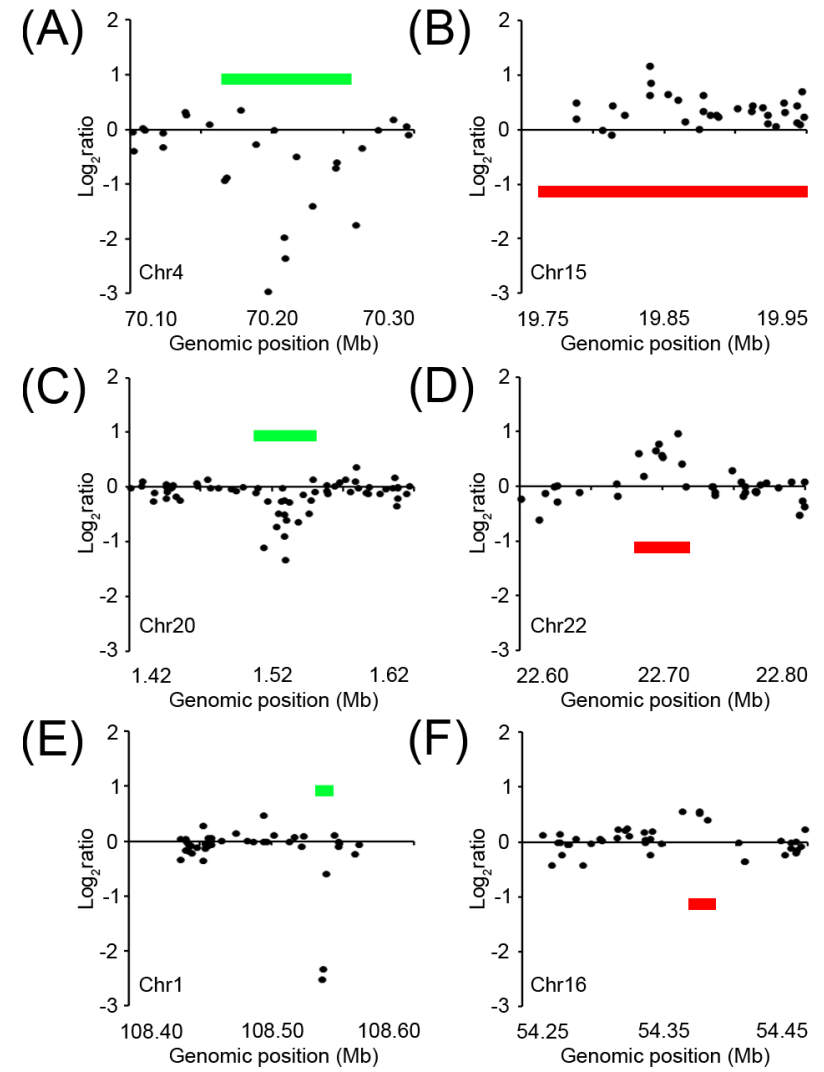

Fig. 1. Signal intensity (Log $R$ ratio) plots of the copy number variation (CNV) regions. (A, B) CNVs detected from all 3 algorithms. (C, D) CNVs detected from 2 of the 3 algorithms. (E, F) CNVs uniquely detected by a single algorithm. Green bars, copy number-loss CNV regions; red bars, copy number-gained CNV regions.

be detected by 1 particular algorithm.

A recent report examining the impact of inaccuracy of $\mathrm{CNV}$ detection from SNP genotyping data revealed that $\mathrm{CNVs}$, defined as the copy number changes of $>7$ consecutive probes, were fairly reliable in case of deletion type $\mathrm{CNVs}$ [29]. In our previous study exploring the CNV profiles of Koreans using an Affymetrix array, we suggested the filtering condition of $\mathrm{CNV}$ call as $>6$ consecutive probes to be reliable [5]. Therefore, to make more reliable conditions in this study, we filtered the $\mathrm{CNV}$ calls as $>7$ consecutive probes. Under this criterion, the number of $\mathrm{CNV}$ calls from the 3 algorithms was reduced down from 295 to 385, while the median size became bigger- $50 \mathrm{~kb}$ by PennCNV and QuantiSNP and $23.5 \mathrm{~kb}$ by BirdSuite (Table 2). In this condition, same as above, the size of the CNVs consistently detected by 2 or more algorithms was generally bigger than that of the CNVs detected by a single method (Table 3).

In terms of the number of CNVs consistently identified by different algorithms, of the $\mathrm{CNV}$ calls using the PennCNV algorithm, $48.6 \%$ (396/814 CNVs) was detected by 2 or more algorithms, and only 4.9\% (40/814 CNVs) was detected by all 3 algorithms. Similarly, of the CNV calls using QuantiSNP, $42.9 \%$ (466/1,087 CNVs) was detected by 2 or more algorithms and only 3.9\% (42/1,087 CNVs) was detected by all 3 algorithms. However, in the case of the BirdSuite algorithm, although the number of $\mathrm{CNV}$ calls was the biggest, only $9.6 \%(131 / 1,362 \mathrm{CNVs})$ was detected by 2 or more algorithms and $2.9 \%(40 / 1,362 \mathrm{CNVs})$ was detected by all 3 algorithms. Taken together, only $17 \%$ of the total CNV calls (465/2,735 CNVs) were defined by more than 2 algorithms, and only $1.5 \%$ (40/2,735 CNVs) was defined by all 3 algorithms (Table 3 ). In the filtering condition of $>7$ consecutive probes, the number of CNVs consistently identified by different algorithms was improved. For example, of the CNV calls using the PennCNV algorithm, $76.6 \%$ (226/295 CNVs) was detected by 2 or more algorithms and only $23.4 \%$ (69/295 CNVs) was detected uniquely by PennCNV. QuantiSNP calls showed a similar trend. However, even under this condition, 92.5\% (356/385 $\mathrm{CNVs}$ ) of BirdSuite calls were unique (Table 3). All the CNVs consistently detected by 2 algorithms were from PennCNV and QuantiSNP, while no CNVs that were detected by the BirdSuite algorithm were consistently detected by 2 algorithms, with only 1 exception. These data suggest that CNV calls from SNP genotyping depend substantially on the characteristics of calling algorithms, and only part of the $\mathrm{CNV}$ calls from each algorithm seems to be reliable. Therefore, CNVs identified from SNP genotyping data must be validated as completely as possible, and especially in the case of using a single calling algorithm, the $\mathrm{CNVs}$ must be validated more carefully. Filtering the CNV 
Table 4. Genomic qPCR validation of the CNV calls from SNP genotyping data

\begin{tabular}{|c|c|c|c|c|c|c|}
\hline \multirow{2}{*}{$\begin{array}{c}\text { Subject } \\
\text { no. }\end{array}$} & \multicolumn{2}{|c|}{ Unique } & \multicolumn{2}{|c|}{2 overlaps } & \multicolumn{2}{|c|}{3 overlaps } \\
\hline & $\begin{array}{l}\mathrm{CNV} \\
\text { call }\end{array}$ & qPCR & $\begin{array}{l}\mathrm{CNV} \\
\text { call }\end{array}$ & qPCR & $\begin{array}{l}\text { CNV } \\
\text { call }\end{array}$ & qPCR \\
\hline \multicolumn{7}{|c|}{ No filtration } \\
\hline 1 & 9 & 3 & 2 & 1 & 0 & 0 \\
\hline 2 & 0 & 0 & 3 & 2 & 1 & 1 \\
\hline 3 & 4 & 1 & 4 & 4 & 0 & 0 \\
\hline 4 & 7 & 3 & 2 & 2 & 3 & 2 \\
\hline 5 & 4 & 1 & 4 & 3 & 0 & 0 \\
\hline 6 & 3 & 3 & 5 & 4 & 0 & 0 \\
\hline 7 & 4 & 2 & 3 & 2 & 1 & 1 \\
\hline 8 & 6 & 3 & 5 & 1 & 1 & 1 \\
\hline 9 & 4 & 1 & 5 & 0 & 0 & 0 \\
\hline \multirow[t]{2}{*}{10} & 6 & 1 & 0 & 0 & 1 & 0 \\
\hline & 47 & $\begin{array}{c}18 \\
(38.3 \%)\end{array}$ & 33 & $\begin{array}{c}19 \\
(57.6 \%)\end{array}$ & 7 & $\begin{array}{c}5 \\
(71.4 \%)\end{array}$ \\
\hline \multicolumn{7}{|c|}{$>7$ consecutive proves } \\
\hline 1 & 6 & 3 & 1 & 1 & 0 & 0 \\
\hline 2 & 0 & 0 & 3 & 2 & 1 & 1 \\
\hline 3 & 1 & 1 & 4 & 4 & 0 & 0 \\
\hline 4 & 3 & 2 & 1 & 1 & 3 & 2 \\
\hline 5 & 2 & 0 & 3 & 3 & 0 & 0 \\
\hline 6 & 0 & 0 & 5 & 4 & 0 & 0 \\
\hline 7 & 1 & 1 & 3 & 2 & 1 & 1 \\
\hline 8 & 2 & 2 & 5 & 1 & 1 & 1 \\
\hline 9 & 3 & 1 & 4 & 0 & 0 & 0 \\
\hline \multirow[t]{2}{*}{10} & 5 & 1 & 0 & 0 & 0 & 0 \\
\hline & 23 & $\begin{array}{c}11 \\
(47.8 \%)\end{array}$ & 29 & $\begin{array}{c}18 \\
(62.1 \%)\end{array}$ & 6 & $\begin{array}{c}5 \\
(83.3 \%)\end{array}$ \\
\hline
\end{tabular}

qPCR, number of consistently detected $\mathrm{CNVs}$ by quantitative PCR; CNV call, number of copy number variation calls; SNP, single-nucleotide polymorphism.

calls by the number of consecutive probes can improve the reliability of $\mathrm{CNV}$ calls.

To validate the CNVs identified from 3 different algorithms in this study, we randomly selected $25 \mathrm{CNV}$ regions across the whole chromosomes. The genomic qPCR validation results are listed in Table 4. In case of the CNVs identified by all 3 algorithms, $71.4 \%$ (5/7) of the consistency was observed between the $\mathrm{CNV}$ call and genomic qPCR result. Of the CNVs identified by more than 2 algorithms, $57.6 \%(19 / 33)$ was consistent. Of the unique CNV calls defined by just a single algorithm, only $38.3 \%$ (18/47) was consistent, but the other $61.7 \%$ (28/46) was not consistent. In the case of the CNVs defined as $>7$ consecutive probes, the consistency was generally improved- $47.8 \%$ (unique CNVs), $62.2 \%$ (CNVs identified by more than 2 algorithms), and $83.3 \%$ (CNVs identified by all 3 algorithms). These results indicate that $\mathrm{CNV}$ calls from 2 or more algorithms are more reliable than those from single algorithms. $\mathrm{CNV}$ calls from all 3 algorithms are, of course, the most reliable, but this is too stringent; so, the number of CNVs is not applicable for GWAS analysis.

In this study, we aimed to verify the effect of adopting multiple $\mathrm{CNV}$ calling algorithms and set up the most reliable pipeline for $\mathrm{CNV}$ calling with Affymetrix Genomewide SNP 5.0 data. We found that CNVs defined by a single CNV calling algorithm may not reliable enough for further GWAS study, regardless of the types of algorithms. Based on our findings, we propose that for reliable CNV-based GWAS using SNP array data, CNV calls must be performed with at least 3 different algorithms, and the CNVs consistently called from more than 2 methods must be used for association analysis.

\section{Acknowledgments}

This study was supported by a grant from the Korea Healthcare Technology R\&D Project (A092258) and the Korea Health 21 R\&D Project (A040002), Ministry of Health and Welfare, Republic of Korea.

\section{References}

1. McCarroll SA, Altshuler DM. Copy-number variation and association studies of human disease. Nat Genet 2007;39(7 Suppl):S37-S42.

2. Feuk L, Carson AR, Scherer SW. Structural variation in the human genome. Nat Rev Genet 2006;7:85-97.

3. Estivill X, Armengol L. Copy number variants and common disorders: filling the gaps and exploring complexity in genome-wide association studies. PLoS Genet 2007;3:17871799.

4. Redon R, Ishikawa S, Fitch KR, Feuk L, Perry GH, Andrews $\mathrm{TD}$, et al. Global variation in copy number in the human genome. Nature 2006;444:444-454.

5. Yim SH, Kim TM, Hu HJ, Kim JH, Kim BJ, Lee JY, et al. Copy number variations in East-Asian population and their evolutionary and functional implications. Hum Mol Genet 2010; 19:1001-1008.

6. Kim JH, Jung SH, Hu HJ, Yim SH, Chung YJ. Comparison of the Affymetrix SNP Array 5.0 and oligoarray platforms for defining CNV. Genomics Inform 2010;8:138-141.

7. Lai WR, Johnson MD, Kucherlapati R, Park PJ. Comparative analysis of algorithms for identifying amplifications and deletions in array CGH data. Bioinformatics 2005;21:3763-3770.

8. Dellinger AE, Saw SM, Goh LK, Seielstad M, Young TL, Li YJ. Comparative analyses of seven algorithms for copy number variant identification from single nucleotide polymorphism arrays. Nucleic Acids Res 2010;38:e105.

9. Barnes C, Plagnol V, Fitzgerald T, Redon R, Marchini J, Clayton D, et al. A robust statistical method for case-control association testing with copy number variation. Nat Genet 2008;40: 1245-1252. 
10. Forer L, Schönherr S, Weissensteiner H, Haider F, Kluckner T, Gieger C, et al. CONAN: copy number variation analysis software for genome-wide association studies. BMC Bioinformatics 2010;11:318.

11. Pique-Regi R, Cáceres A, González JR. R-Gada: a fast and flexible pipeline for copy number analysis in association studies. BMC Bioinformatics 2010;11:380.

12. Purcell S, Neale B, Todd-Brown K, Thomas L, Ferreira MA, Bender D, et al. PLINK: a tool set for whole-genome association and population-based linkage analyses. Am J Hum Genet 2007;81:559-575.

13. Subirana I, Diaz-Uriarte R, Lucas G, Gonzalez JR. CNVassoc: association analysis of CNV data using R. BMC Med Genomics 2011;4:47.

14. Kim JH, Hu HJ, Yim SH, Bae JS, Kim SY, Chung YJ. CNVRuler: a copy number variation-based case-control association analysis tool. Bioinformatics 2012;28:1790-1792.

15. Winchester L, Yau C, Ragoussis J. Comparing CNV detection methods for SNP arrays. Brief Funct Genomic Proteomic 2009;8: 353-366.

16. Kidd JM, Cooper GM, Donahue WF, Hayden HS, Sampas N, Graves T, et al. Mapping and sequencing of structural variation from eight human genomes. Nature 2008;453:56-64.

17. Baumbusch LO, Aarøe J, Johansen FE, Hicks J, Sun H, Bruhn L, et al. Comparison of the Agilent, ROMA/NimbleGen and Illumina platforms for classification of copy number alterations in human breast tumors. BMC Genomics 2008;9:379.

18. Curtis C, Lynch AG, Dunning MJ, Spiteri I, Marioni JC, Hadfield J, et al. The pitfalls of platform comparison: DNA copy number array technologies assessed. BMC Genomics 2009;10:588.

19. Hester SD, Reid L, Nowak N, Jones WD, Parker JS, Knudtson $\mathrm{K}$, et al. Comparison of comparative genomic hybridization technologies across microarray platforms. J Biomol Tech 2009; 20:135-151.

20. Pinto D, Darvishi K, Shi X, Rajan D, Rigler D, Fitzgerald T, et al. Comprehensive assessment of array-based platforms and calling algorithms for detection of copy number variants. Nat
Biotechnol 2011;29:512-520.

21. Ramayo-Caldas Y, Castelló A, Pena RN, Alves E, Mercadé A, Souza CA, et al. Copy number variation in the porcine genome inferred from a 60 k SNP BeadChip. BMC Genomics 2010; 11:593.

22. Degenhardt F, Priebe L, Herms S, Mattheisen M, Mühleisen TW, Meier S, et al. Association between copy number variants in 16p11.2 and major depressive disorder in a German case-control sample. Am J Med Genet B Neuropsychiatr Genet 2012;159B:263-273.

23. Marenne G, Rodríguez-Santiago B, Closas MG, Pérez-Jurado L, Rothman N, Rico D, et al. Assessment of copy number variation using the Illumina Infinium 1M SNP-array: a comparison of methodological approaches in the Spanish Bladder Cancer/EPICURO study. Hum Mutat 2011;32:240-248.

24. Kawamura Y, Otowa T, Koike A, Sugaya N, Yoshida E, Yasuda $\mathrm{S}$, et al. A genome-wide $\mathrm{CNV}$ association study on panic disorder in a Japanese population. J Hum Genet 2011;56: 852-856.

25. Wang K, Li M, Hadley D, Liu R, Glessner J, Grant SF, et al. PennCNV: an integrated hidden Markov model designed for high-resolution copy number variation detection in whole-genome SNP genotyping data. Genome Res 2007;17:1665-1674.

26. Colella S, Yau C, Taylor JM, Mirza G, Butler H, Clouston P, et al. QuantiSNP: an Objective Bayes Hidden-Markov Model to detect and accurately map copy number variation using SNP genotyping data. Nucleic Acids Res 2007;35:2013-2025.

27. Korn JM, Kuruvilla FG, McCarroll SA, Wysoker A, Nemesh J, Cawley $\mathrm{S}$, et al. Integrated genotype calling and association analysis of SNPs, common copy number polymorphisms and rare CNVs. Nat Genet 2008;40:1253-1260.

28. Yim SH, Chung YJ, Jin EH, Shim SC, Kim JY, Kim YS, et al. The potential role of VPREB1 gene copy number variation in susceptibility to rheumatoid arthritis. Mol Immunol 2011;48: 1338-1343.

29. Wineinger NE, Tiwari HK. The impact of errors in copy number variation detection algorithms on association results. PLoS One 2012;7:e32396. 